\title{
Structure of Applicable Surfaces from Single Views
}

\author{
Nail Gumerov, Ali Zandifar, Ramani Duraiswami and Larry S. Davis \\ Perceptual Interfaces and Reality Lab, University of Maryland, College Park \\ \{gumerov, alizand, ramani, lsd\}@umiacs.umd.edu
}

\begin{abstract}
The deformation of applicable surfaces such as sheets of paper satisfies the differential geometric constraints of isometry (lengths and areas are conserved) and vanishing Gaussian curvature. We show that these constraints lead to a closed set of equations that allow recovery of the full geometric structure from a single image of the surface and knowledge of its undeformed shape. We show that these partial differential equations can be reduced to the Hopf equation that arises in non-linear wave propagation, and deformations of the paper can be interpreted in terms of the characteristics of this equation. A new exact integration of these equations is developed that relates the 3-D structure of the applicable surface to an image. The solution is tested by comparison with particular exact solutions. We present results for both the forward and the inverse 3D structure recovery problem.
\end{abstract}

Keywords: Surface, Differential Geometry, Applicable Surfaces, Shape from $X$

\section{Introduction}

When a picture or text printed on paper is imaged, we are presented with a problem of unwarping the captured digital image to its flat, fronto-parallel representation, as a preprocessing step before performing tasks such as identification, or Optical Character Recognition (OCR). In the case that the paper is flat, the problem reduces to one of undoing a projection of an initial shape such as a rectangle, and the rectification (or unwarping) can be achieved by computing a simple homography. A harder problem is when the piece of paper is itself deformed or bent. In this case the unwarping must undo both the effects of the three-dimensional bending of the surface, and the imaging process. The differential geometry of surfaces provides a very powerful set of relations for analysis of the unwarping. However, most quantitative use of differential geometry has been restricted to range data, while its use for image data has been primarily qualitative. The deformation of paper surfaces satisfies the conditions of isometry and vanishing Gaussian curvature. Here, we show that these conditions can be analytically integrated to infer the complete 3D structure of the surface from an image of its bounding contour.

Previous authors have attempted to enforce these conditions in 3D reconstruction. However, they essentially enforced these as constraints to a process of polynomial/spline fitting using data obtained on the surface [16]. In contrast, we solve these equations, and show that information on the bounding contour is sufficient to determine structure completely. Further, exact correspondence information along the bounding contour is not needed. We only need the correspondences of a few points, e.g., corners. Other than its theoretical importance, our research can potentially benefit diverse computer vision applications, e.g. portable scanning devices, digital flattening of creased documents, 3D reconstruction without correspondence, and perhaps most importantly, OCR of scene text. 


\section{Previous Work}

A seminal paper by Koenderink [7] addressed the understanding of 3D structure qualitatively from occluding contours in images. It was shown that the concavities and convexities of visual contours are sufficient to infer the local shape of a surface. Here, we perform quantitative recovery of 3D surface structure for the case of applicable surfaces. While we were not able to find similar papers dealing with analytical integration of the equations of differential geometry to obtain structure, the following papers deal with related problems of unwarping scene text, or using differential geometric constraints for reconstruction.

Metric rectification of planar surfaces: In $[2,12,15]$ algorithms for performing metric rectification of planar surfaces were considered. These papers extract from the images, features such as vanishing lines and right angles and perform rectification. Extraction of vanishing lines is achieved by different methods; such as the projection profile method [2] and the illusory and non-illusory lines in textual layouts [15].

Undoing paper curl for non-planar surfaces knowing range data: A number of papers deal with correcting the curl of documents using known shape (e.g. cylinders) $[11,19]$. These approaches all need 3D points on the surface to solve for the inverse mapping. In [16] sparse 3D data on the curled paper surface was obtained from a laser device. An approximate algorithm to fit an applicable surface through these points was developed that allowed obtaining dense depth data. The isometry constraint was approximately enforced by requiring that distances between adjacent nodes be constant. In [1] a mass-spring particle system framework was used for digital flattening of destroyed documents using depth measurements, though the differential geometry constraints are not enforced.

Isometric surfaces: In [10] an algorithm is developed to bend virtual paper without shearing or tearing. Ref. [13] considers the shape-from-motion problem for shapes deformed under isometric mapping.

\section{Theory}

\subsection{Basic Surface Representation}

A surface is the exterior boundary of an object/body. In a 3D world coordinate system, a surface $\mathbf{r}=\mathbf{r}(X, Y, Z)$, (where $(X, Y, Z)$ is any point on the surface) is mathematically represented in explicit, implicit and parametric forms respectively as:

$$
z=f(x, y), \quad F(x, y, z)=0, \quad \mathbf{r}(u, v)=(X(u, v), Y(u, v), Z(u, v)) .
$$

Consider a smooth surface $S$ expressed parametrically as:

$$
\mathbf{r}(u, v)=(X(u, v), Y(u, v), Z(u, v)),
$$

which is a mapping from any point $(u, v)$ in the parametric (or undeformed) plane (uvplane) to a point $(X, Y, Z)$ on the surface in $3 \mathrm{D}$ (Figure 3). The sets $\{\mathbf{r}(u, v), v=$ const $\}$ and $\{\mathbf{r}(u, v), u=$ const $\}$ represent two families of curves on the surface, whose partial derivatives are tangent vectors to the curves $v=$ const and $u=$ const respectively. These derivatives are often called tangent vectors [9]. Let the second derivatives of $\mathbf{r}$ with respect to $u$ and $v$ be $\mathbf{r}_{u u}, \mathbf{r}_{u v}$ and $\mathbf{r}_{v v}$. The element of distance $d s=|d \mathbf{r}|$ 


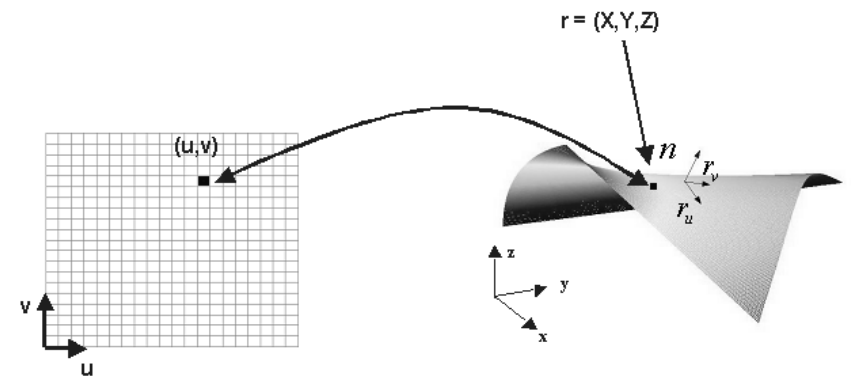

Fig. 1. Parametric representation of a surface

on the surface is given at each surface point $(u, v)$ by the first fundamental form of a surface

$$
\begin{array}{r}
d s^{2}=|d \mathbf{r}|^{2}=\left\|\mathbf{r}_{u}\right\|^{2} d u^{2}+2 \mathbf{r}_{u} \cdot \mathbf{r}_{v} d u d v+\left\|\mathbf{r}_{v}\right\|^{2} d v^{2}=E d u^{2}+2 F d u d v+G d v^{2}, \\
E(u, v)=\left\|\mathbf{r}_{u}\right\|^{2}, \quad F(u, v)=\mathbf{r}_{u} \cdot \mathbf{r}_{v}, \quad G(u, v)=\left\|\mathbf{r}_{v}\right\|^{2} .
\end{array}
$$

The surface coordinates are orthogonal iff $F \equiv 0$. The surface normal $\mathbf{n}$ and area element $d \mathbf{n}$ can be defined in terms of the tangent vectors as:

$$
\mathbf{n}=\frac{\mathbf{r}_{u} \times \mathbf{r}_{v}}{\left|\mathbf{r}_{u} \times \mathbf{r}_{v}\right|}=\sqrt{E G-F^{2}}, \quad d \mathbf{n}=\left|\mathbf{r}_{u} \times \mathbf{r}_{v}\right| d u d v=\sqrt{E G-F^{2}} d u d v .
$$

The second fundamental form of a surface at a point $(u, v)$ measures how far the surface is from being planar. It is given by

$$
-d \mathbf{r} \cdot d \mathbf{n}=L(u, v) d u^{2}+2 M(u, v) d u d v+N(u, v) d v^{2},
$$

where $L, M$ and $N$ are standard and defined e.g., in [9]. For every normal section through $(u, v)$ there exist two principal curvatures $\left(k_{1}, k_{2}\right)$. The mean and Gaussian curvature; $H(u, v)$ and $K(u, v)$ are

$$
H \equiv \frac{k_{1}+k_{2}}{2}=\frac{1}{2} \frac{E N-2 F M+G L}{E G-F^{2}}, \quad K \equiv k_{1} k_{2}=\frac{L N-M^{2}}{E G-F^{2}} .
$$

\subsection{Special Surfaces}

Let us assume that we have a mapping of a point in the parametric plane $(u, v)$ to a point in $3 \mathrm{D}(X, Y, Z)$. The mapping is isometric if the length of a curve or element of area is invariant with the mapping, i.e.

$$
E(u, v)=\left\|\mathbf{r}_{u}\right\|^{2}=1, \quad F(u, v)=\mathbf{r}_{u} \cdot \mathbf{r}_{v}=0, \quad G(u, v)=\left\|\mathbf{r}_{v}\right\|^{2}=1 .
$$

Lengths and areas are conserved in an isometric mapping

$$
\begin{array}{r}
d s^{2}=|d \mathbf{r}|^{2}=E(u, v) d u^{2}+2 F(u, v) d u d v+G(u, v) d v^{2}=d u^{2}+d v^{2}, \\
d A=\sqrt{E G-F^{2}} d u d v=d u d v .
\end{array}
$$


The mapping is conformal if the angle between curves on a surface is invariant of the mapping $(F=0)$. It is developable if the Gaussian curvature is zero everywhere.

$$
K=0 \Longrightarrow L N-M^{2}=0 .
$$

It is applicable if the surface is isometric with a flat surface (Eq. 6) and the Gaussian curvature vanishes (Eq. 7) for every point on the surface.

\subsection{Differential Equations for Applicable Surfaces}

If we differentiate Eq. (6), we have:

$$
\mathbf{r}_{u u} \cdot \mathbf{r}_{u}=\mathbf{r}_{u u} \cdot \mathbf{r}_{v}=\mathbf{r}_{u v} \cdot \mathbf{r}_{u}=\mathbf{r}_{u v} \cdot \mathbf{r}_{v}=\mathbf{r}_{v v} \cdot \mathbf{r}_{u}=\mathbf{r}_{v v} \cdot \mathbf{r}_{v}=0 .
$$

This shows that $\mathbf{r}_{u u}=\left(X_{u u}, Y_{u u}, Z_{u u}\right), \mathbf{r}_{u v}=\left(X_{u v}, Y_{u v}, Z_{u v}\right)$ and $\mathbf{r}_{v v}=\left(X_{v v}, Y_{v v}, Z_{v v}\right)$ are perpendicular to $\mathbf{r}_{u}$ and $\mathbf{r}_{v}$ and consequently, are collinear with the normal vector to the surface.

$$
\mathbf{n}\left\|\left(\mathbf{r}_{u} \times \mathbf{r}_{v}\right)\right\| \mathbf{r}_{u u}\left\|\mathbf{r}_{u v}\right\| \mathbf{r}_{v v},
$$

where $\|$ denotes "is parallel to". We can thus express $\mathbf{n}$ as

$$
\mathbf{n}=a \mathbf{r}_{u u}=b \mathbf{r}_{u v}=c \mathbf{r}_{v v} .
$$

We can rewrite (7) using (10) as:

$$
L N-M^{2}=0 \Longrightarrow a\|\mathbf{n}\|^{2} c\|\mathbf{n}\|^{2}-b^{2}\|\mathbf{n}\|^{2}\|\mathbf{n}\|^{2}=0 \quad \Longrightarrow a c-b^{2}=0,
$$

where $a, b$, and $c$ are scalars, and

$$
\frac{\mathbf{r}_{u v}}{\mathbf{r}_{u u}}=\frac{a}{b}=\frac{b}{c}=\frac{\mathbf{r}_{v v}}{\mathbf{r}_{u v}} .
$$

Therefore from (12) we have:

$$
\frac{\partial^{2} W}{\partial v^{2}} \frac{\partial^{2} W}{\partial u^{2}}=\left(\frac{\partial^{2} W}{\partial u \partial v}\right)^{2}, \text { for } W=X, Y, Z .
$$

Solving the set of nonlinear higher order partial differential equations (PDEs) (Eq. 13), we can compute the surface structure $\mathbf{r}$ in 3D, given boundary conditions (curves) for an applicable surface. These equations may be solved by conventional methods of solving PDEs e.g. Finite Differences or FEM. However, we provide a much more efficient method, based on reducing the solution to integration of several simultaneous ODEs.

\subsection{A First Integration: Reduction to ODEs}

Let $W_{u}=\partial W / \partial u, W_{v}=\partial W / \partial v$. The functions $W_{u}(u, v)$ and $W_{v}(u, v)$ satisfy the consistency conditions

$$
\frac{\partial W_{u}}{\partial v}=\frac{\partial W_{v}}{\partial u}, \quad W=X, Y, Z .
$$

i.e. cross-derivatives are the same. From Eqs. (13) and (14) we have

$$
\frac{\partial W_{u}}{\partial u} \frac{\partial W_{v}}{\partial v}-\frac{\partial W_{u}}{\partial v} \frac{\partial W_{v}}{\partial u}=\frac{\partial\left(W_{u}, W_{v}\right)}{\partial(u, v)}=0 .
$$


Therefore Eq. (13) can be treated as a degeneracy condition for the Jacobian of the mapping from $(u, v) \longmapsto\left(W_{u}, W_{v}\right)$. This degeneracy means that the functions $W_{u}$ and $W_{v}$ are functions of a single variable, $t$, which in turn is a function of $(u, v)$. In other words:

$$
\exists t=t(u, v) \text { such that } W_{u}(u, v)=W_{u}(t), \quad W_{v}(u, v)=W_{v}(t),
$$

where $W=X, Y, Z$. In this case $t=$ const is a line in the parametric plane. Since $W$ denotes any of $X, Y$ and $Z$, Eq. (16) could hold separately for each component, with some different mapping functions $t_{x}(u, v), t_{y}(u, v)$, and $t_{z}(u, v)$ specific to each coordinate. However, these functions must all be equal because all are functions of the single variable $t(u, v)$, which can be called the mapping or characteristic function for the surface $S$. Therefore,

$$
\mathbf{r}_{u}=\mathbf{r}_{u}(t), \quad \mathbf{r}_{v}=\mathbf{r}_{v}(t)
$$

where $t=t(u, v)$. Denoting by the superscript dot the derivative of a function with respect to $t$, we can write $\mathbf{r}_{u u}$ and $\mathbf{r}_{v v}$ as

$$
\mathbf{r}_{u u}=\dot{\mathbf{r}}_{u} \frac{\partial t}{\partial u}, \quad \mathbf{r}_{v v}=\dot{\mathbf{r}}_{v} \frac{\partial t}{\partial v}
$$

From Eq. $(9,18)$, we see that $\dot{\mathbf{r}}_{u}$ and $\dot{\mathbf{r}}_{v}$ are collinear with the surface normal i.e. $\dot{\mathbf{r}}_{u}\left\|\mathbf{n}, \dot{\mathbf{r}}_{v}\right\| \mathbf{n}$. Let us define a new vector $\mathbf{w}$ as :

$$
\mathbf{w}=u \dot{\mathbf{r}}_{u}(t)+v \dot{\mathbf{r}}_{v}(t) .
$$

Also note that $\mathbf{w}$ is a function of the characteristic variable $t$, since the Jacobian of a mapping from $(u, v) \longmapsto(t, \mathbf{m} \cdot w)$ for a constant vector $\mathbf{m}$ vanishes:

$$
\begin{array}{r}
\frac{\partial(t, \mathbf{w} \cdot \mathbf{m})}{\partial(u, v)}=\frac{\partial t}{\partial u} \frac{\partial \mathbf{w} \cdot \mathbf{m}}{\partial v}-\frac{\partial t}{\partial v} \frac{\partial \mathbf{w} \cdot \mathbf{m}}{\partial u}=\frac{\partial t}{\partial u} \dot{\mathbf{r}}_{v}(t) \cdot \mathbf{m}-\frac{\partial t}{\partial u} \dot{\mathbf{r}}_{\mathbf{v}}(\mathbf{t}) \cdot \mathbf{m} \\
=\mathbf{r}_{u v} \cdot \mathbf{m}-\mathbf{r}_{u v} \cdot \mathbf{m} \Longrightarrow \frac{\partial(t, \mathbf{w} \cdot \mathbf{m})}{\partial(u, v)}=0 .
\end{array}
$$

This means that $\mathbf{w}$ is a function of $t$ alone; $\mathbf{w}=\mathbf{w}(t)$. From collinearity of $\mathbf{w}$ with $\dot{\mathbf{r}}_{u}$ and $\dot{\mathbf{r}}_{v}$ it follows that two scalar functions $h_{u}(t)$ and $h_{v}(t)$ can be introduced as

$$
\dot{\mathbf{r}}_{u}(t)=h_{u}(t) \mathbf{w}(t), \quad \dot{\mathbf{r}}_{v}(t)=h_{v}(t) \mathbf{w}(t) .
$$

By (20), and from Eq. (19), we have

$$
u h_{u}(t)+v h_{v}(t)=1, \quad h_{v}(t) \dot{\mathbf{r}}_{u}(t)-h_{u}(t) \dot{\mathbf{r}}_{v}(t)=0 .
$$

Therefore, Eq.(21) defines a characteristic line in the $u v$-plane for $t=$ const. While the latter equation provides a relation between functions of $t$, the former implicitly determines $t(u, v)$. Since $h_{u}(t)$ and $h_{v}(t)$ are known, Eq. (21) gives $t(u, v)$. Note that $t$ satisfies the equation

$$
h_{v}(t) \frac{\partial t}{\partial u}-h_{u}(t) \frac{\partial t}{\partial v}=0,
$$


which is a Hopf-type equation, a common nonlinear hyperbolic equation in shock-wave theory [4]. The characteristics of this equation are $t(u, v)$ which satisfies

$$
t(u, v)=t(u+c(t) v), \quad c(t)=\frac{h_{u}(t)}{h_{v}(t)} .
$$

Therefore, for any $t=$ const the characteristic is a line in the $u v$-plane. The properties of the Hopf equation are well studied in the theory of propagation of shock waves in nonlinear media ([4]). Along the characteristics, $t=$ const, all functions of $t$ are constant, including $h_{u}(t)$ and $h_{v}(t)$. As follows from Eq. (21), in the $(u, v)$-plane these characteristics are straight lines. The lines corresponding to characteristics are also straight lines on the surface. In fact to generate an applicable surface, we can sweep a line in space and the generated envelope will be applicable. Through every point on

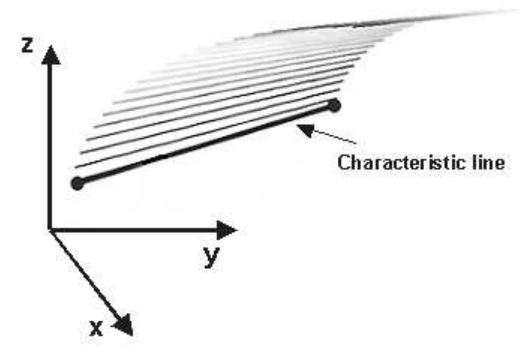

Fig. 2. Characteristics lines as generator lines

the surface there is a straight line as shown (Figure 2) by:

$$
\mathbf{r}(t)=u \mathbf{r}_{u}(t)+v \mathbf{r}_{v}(t)+\rho(t) \quad, \quad \dot{\rho}(t)=-\mathbf{w}(t),
$$

The above equations are sufficient to solve the basic warping and unwarping problems for images based on information about the shapes of the image boundaries. The goal is to find for any characteristic line, the variables $\mathbf{r}_{u}(t), \mathbf{r}_{v}(t), \rho(t), h_{u}(t)$ and $h_{v}(t)$ and, finally, $\mathbf{r}(t)$ from available information. To summarize the differential and algebraic relations for applicable surfaces, we have

$$
\begin{gathered}
\mathbf{r}(u, v)=u \mathbf{r}_{u}(t)+v \mathbf{r}_{v}(t)+\rho(t), \quad \dot{\mathbf{r}}_{u}(t)=h_{u}(t) \mathbf{w}(t), \dot{\mathbf{r}}_{v}(t)=h_{v}(t) \mathbf{w}(t), \\
\dot{\rho}(t)=-\mathbf{w}(t), \quad u h_{u}(t)+v h_{v}(t)=1,\left\|\mathbf{r}_{u}\right\|^{2}=1, \quad \mathbf{r}_{u} \cdot \mathbf{r}_{v}=0, \quad\left\|\mathbf{r}_{v}\right\|^{2}=1 .
\end{gathered}
$$

\subsection{Forward Problem: Surface with a Specified Boundary Curve}

Here, we specify the bending of a flat page in 3D so that one edge conforms to a given $3 \mathrm{D}$ curve. We call this the forward problem. We generate the warped surface to demonstrate the solution to Eq. (25). Let $\Gamma^{\prime}$ be an open curve on a patch $\Omega^{\prime} \subset P$ in the $u v$-plane, corresponding to an open curve $\Gamma$ in 3D. To generate an applicable surface in 3D, knowledge of the corresponding curves $\Gamma^{\prime}$ and $\Gamma$ and the patch boundaries in the $u v$-plane (Figure 3 ) are sufficient. We know that the curve $\Gamma^{\prime}$ starts from a point $A^{\prime}=\left(u_{0}, v_{0}\right)$ and the corresponding curve $\Gamma$ passes from $A=\left(X_{0}, Y_{0}, Z_{0}\right)$ and the 


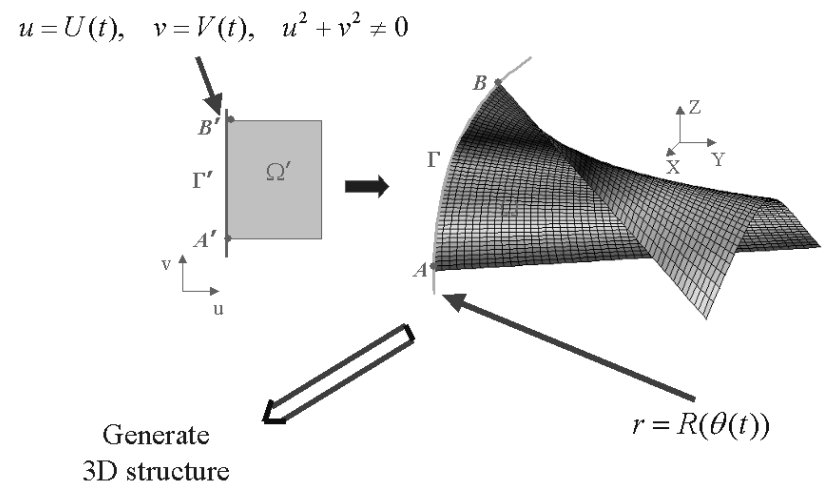

Fig. 3. Generation of an applicable surface with a 3D curve. In this example a straight line $\Gamma^{\prime}$ in the $u v$-plane is mapped on a given $3 \mathrm{D}$ curve $\Gamma$.

point $B$ corresponds to the point $B^{\prime}$. Due to isometry, the length of the two curves are the same, and there is a one-to-one mapping from a domain $\Omega^{\prime} \subset P$ to $\Omega \subset S$, which are respectively bounded by $\Gamma^{\prime}$ and $\Gamma$. For any point $\left(u^{*}, v^{*}\right) \in \Omega^{\prime}$ there exists a characteristic, $t=t^{*}$, which also passes through some point on $\Gamma^{\prime}$. Assume now that $\Gamma^{\prime}$ is specified by the parametric equations

$$
u=U(t), \quad v=V(t), \quad u^{2}+v^{2} \neq 0 .
$$

Without loss of generality, we can select $t$ to be a natural parameterization of $\Gamma^{\prime}$, measured from point $A^{\prime}$; i.e. the arc length $s$ along the curve $\Gamma$, measured from the curve starting point $t=t_{0}$,

$$
s \equiv \int_{t_{0}}^{t} d s \equiv \int_{t_{0}}^{t} \sqrt{d \mathbf{r} \cdot d \mathbf{r}} .
$$

parametrizes the curve. Let $\Gamma^{\prime}:(U(t), V(t))$ be in $\left[t_{\min }, t_{\max }\right]$. If we represent $\Gamma$ in parametric form as $\mathbf{r}=\mathbf{R}(t)$, then due to isometry, $t$ will also be a natural parameter for $\Gamma^{\prime}$, and

$$
\dot{U}^{2}+\dot{V}^{2}=1, \quad \dot{\mathbf{R}} \cdot \dot{\mathbf{R}}=1 .
$$

The surface equations for any $(u, v) \in \Omega^{\prime}$ are

$$
\begin{gathered}
\mathbf{r}_{u} \cdot \mathbf{r}_{u}=1, \quad \mathbf{r}_{u} \cdot \mathbf{r}_{v}=0, \quad \mathbf{r}_{v} \cdot \mathbf{r}_{v}=1 \\
U h_{u}+V h_{v}=1, \quad h_{v} \dot{\mathbf{r}}_{u}-h_{u} \dot{\mathbf{r}}_{v}=\mathbf{0}, \quad U \mathbf{r}_{u}+V \mathbf{r}_{v}+\rho=\mathbf{R} .
\end{gathered}
$$

While the number of unknowns here is $11\left(\mathbf{r}_{u}, \mathbf{r}_{v}, \rho, h_{u}, h_{v}\right)$ and the number of equations are 12 (Eqs. 27,28) but two of them are dependent(Eqs. including $h_{u}$ and $h_{v}$ ). For unique solution of Eqs. $(27,28)$, we differentiate Eq. (27) to obtain sufficient equations to solve the forward problem

$$
\begin{gathered}
\dot{\mathbf{r}}_{u}=\frac{h_{u} \mathbf{F}}{\dot{U} h_{u}+\dot{V} h_{v}}, \dot{\mathbf{r}}_{v}=\frac{h_{v} \mathbf{F}}{\dot{U} h_{u}+\dot{V} h_{v}}, h_{u}=\frac{g_{u}}{V g_{v}+U g_{u}}, \quad h_{v}=\frac{g_{v}}{V g_{v}+U g_{u}} \\
\mathbf{F}=\ddot{\mathbf{R}}-\ddot{U} \mathbf{r}_{u}-\ddot{V} \mathbf{r}_{v}, g_{u}=\dddot{U}-\dddot{\mathbf{R}} \cdot \mathbf{r}_{u}, g_{v}=\dddot{V}-\dddot{\mathbf{R}} \cdot \mathbf{r}_{v}
\end{gathered}
$$


These equations must be integrated numerically using, e.g., the Runge-Kutta method [17]. To generate the structure of the applicable surface we need for any characteristic line, the functions $\mathbf{r}_{u}(t), \mathbf{r}_{v}(t)$ and $\rho(t) ;\left(\mathbf{r}_{u}(t), \mathbf{r}_{v}(t)\right)$ are obtained from the solution to ODEs, while $\rho(t)$ is computed from the fifth equation in (28). The solution to our problem is a two-point boundary value problem (bvp). Most software for ODEs is written for initial value problems. To solve a bvp using an initial value solver, we need to estimate $\mathbf{r}_{u 0}=\mathbf{r}_{u}(0)$ and $\mathbf{r}_{v 0}=\mathbf{r}_{v}(0)$. which achieves the correct boundary value. The vectors $\mathbf{r}_{u 0}$ and $\mathbf{r}_{v 0}$ are dependent, since they satisfy the first three equations (28), which describe two orthonormal vectors. Assuming that $\left(\mathbf{r}_{u}, \mathbf{r}_{v}, \mathbf{r}_{u} \times \mathbf{r}_{v}\right)$ is a right-handed basis, we can always rotate the reference frame of the world coordinates so that in the rotated coordinates we have $\mathbf{r}_{u 0}=(1,0,0), \mathbf{r}_{v 0}=(0,1,0)$. Consistent initial conditions $\mathbf{r}_{u 0}$ and $\mathbf{r}_{v 0}$ for Eq. (28) can be obtained by application of a rotation matrix $Q(\alpha, \beta, \gamma)$ with Euler angles $\alpha, \beta$ and $\gamma$, to the vectors $(1,0,0)$ and $(0,1,0)$, respectively. We also can note that for some particular cases it may happen that both the functions $g_{v}$ and $g_{u}$ in Eq. (29) may be zero. In this case the equations for $h_{u}$ and $h_{v}$ can be replaced by the limiting expressions for $g_{v} \rightarrow 0, g_{u} \rightarrow 0$. In the special case (rectangular patch in the parametric plane), we can show that there is an analytical solution given by:

$$
\mathbf{r}_{u}=\frac{\ddot{\mathbf{R}} \times \dot{\mathbf{R}}}{|\ddot{\mathbf{R}}|}, \quad \mathbf{r}_{v}=\dot{\mathbf{R}} .
$$

\subsection{Inverse Problem: 3D Structure Recovery of Applicable Surfaces}

Here, we seek to estimate the 3D structure of an applicable surface from a single view (with known camera model) and knowledge of the undeformed $u v$ plane boundary. For

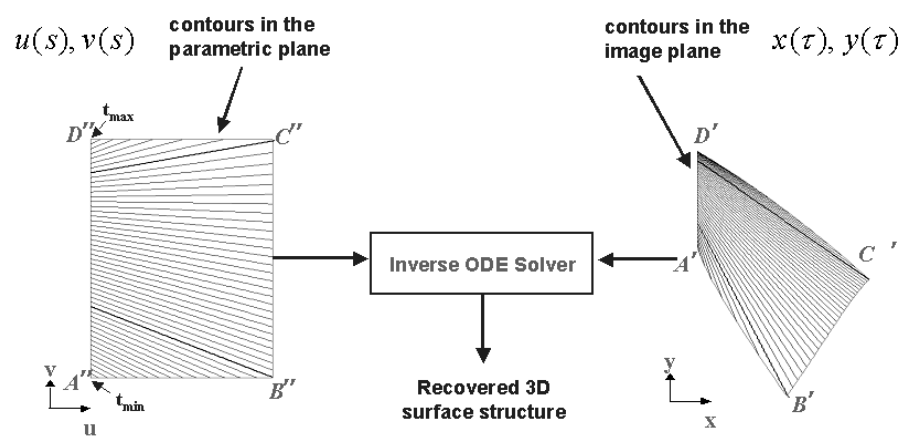

Fig. 4. Inverse Problem Schematic

any point $(x, y)$ in the image plane, we can estimate the corresponding point in the $u v$ plane and vice versa by solving the ODEs for the problem. The input parameters are the known camera model, the patch contours in the $u v$-plane and the image plane. Assume that the image of the patch $\left(\Omega^{\prime}\right)$ is bounded by two curves $\Gamma_{1}^{\prime}$ and $\Gamma_{2}^{\prime}$, the corresponding patch $(\Omega)$ in the $u v$-plane is bounded by $\Gamma_{1}$ and $\Gamma_{2}$ and that the patch $\Omega$ bounded by the two characteristics, $t=t_{\min }$, and $t=t_{\max }$ (Fig. 4). We assume that $\Gamma_{1}$ and $\Gamma_{2}$ are piecewise continuous curves in the $u v$-plane, and not tangential to the characteristic lines $t_{\min }<t<t_{\max }$. For any point $\left(u_{*}, v_{*}\right) \in \Omega$ there exists a characteristic, $t=t_{*}$, 
which passes through some points on $\Gamma_{1}$ and some points on $\Gamma_{2}$. In the $u v$-plane these curves can be specified by a natural parameterization $u=U_{1}\left(s_{1}\right), v=V_{1}\left(s_{1}\right)$ for $\Gamma_{1}$, and $u=U_{2}\left(s_{2}\right), v=V_{2}\left(s_{2}\right)$ for $\Gamma_{2}$, with $u^{2}+v^{2} \neq 0$. Here $s_{1}(t)$ and $s_{2}(t)$ are unknown and must be found in the process of solution.

$\Gamma_{1}$ and $\Gamma_{2}$ correspond to the $3 \mathrm{D}$ curves $\mathbf{r}=\mathbf{r}_{1}(t)$ and $\mathbf{r}=\mathbf{r}_{2}(t)$, which are unknown and found in the process of solution. Note that at the starting point or end point, $\Gamma_{1}$ and $\Gamma_{2}$ may intersect. At such a point the characteristic $t=t_{\min }$ or $t=t_{\max }$ is tangential to the boundary or the boundary is not smooth (e.g. we are at a corner). In case $\Gamma_{1}$ and $\Gamma_{2}$ intersect at $t=t_{\min }$ and $t=t_{\max }$ they completely define the boundary of the patch $\Omega$. These cases are not special and can be handled by the general method described below. Assume that the camera is calibrated, and the relation between the world coordinates $\mathbf{r}=(X, Y, Z)$ and coordinates of the image plane $(x, y)$ are known as $x=F_{x}(\mathbf{r})$ and $y=F_{y}(\mathbf{r})$. What is also known are the equations for $\Gamma_{1}^{\prime}$ and $\Gamma_{2}^{\prime}$ that are images of the patch boundaries $\Gamma_{1}$ and $\Gamma_{2}$. These equations, assumed to be in the form $x=x_{1}\left(\tau_{1}\right), y=y_{1}\left(\tau_{1}\right)$ for $\Gamma_{1}^{\prime}$; and $x=x_{2}\left(\tau_{2}\right), y=y_{2}\left(\tau_{2}\right)$ for $\Gamma_{2}^{\prime}$. Here $\tau_{1}$ and $\tau_{2}$ are the natural parameters of these curves; $\tau_{1}(t)$ and $\tau_{2}(t)$ are obtained from the solution. The specification of the curve parameters as "natural" means:

$$
U_{i}^{\prime 2}+V_{i}^{\prime 2}=1, \quad x_{i}^{\prime 2}+y_{i}^{\prime 2}=1, \quad i=1,2 .
$$

A complete set of equations describing the surface can be reduced then to

$$
\begin{gathered}
\mathbf{r}_{u} \cdot \mathbf{r}_{u}=1, \quad \mathbf{r}_{u} \cdot \mathbf{r}_{v}=0, \quad \mathbf{r}_{v} \cdot \mathbf{r}_{v}=1, \\
\mathbf{r}_{2}=\left(U_{2}-U_{1}\right) \mathbf{r}_{u}+\left(V_{2}-V_{1}\right) \mathbf{r}_{v}+\mathbf{r}_{1}, \quad \dot{\mathbf{r}}_{i}=\dot{s}_{i}\left(U_{i}^{\prime} \mathbf{r}_{u}+V_{i}^{\prime} \mathbf{r}_{v}\right), \\
F_{x}\left(\mathbf{r}_{i}\right)=x_{i}\left(\tau_{i}\right), \quad F_{y}\left(\mathbf{r}_{i}\right)=y_{i}\left(\tau_{i}\right), \quad i=1,2 .
\end{gathered}
$$

We have 16 equations relating the 15 unknowns $\left(\mathbf{r}_{u}, \mathbf{r}_{v}, \mathbf{r}_{1}, \mathbf{r}_{2}, s_{1}, s_{2}, \tau_{1}, \tau_{2}\right)$. As in the previous case, one equation depends the other 15 and so the system is consistent. After $s(t), \mathbf{r}_{1}(t), \mathbf{r}_{u}(t)$, and $\mathbf{r}_{v}(t)$ are found, $h_{u}, h_{v}$, and $\rho$ can be determined as

$$
h_{u}=\frac{V_{2}-V_{1}}{U_{1} V_{2}-U_{2} V_{1}}, \quad h_{v}=\frac{U_{1}-U_{2}}{U_{1} V_{2}-U_{2} V_{1}}, \rho=\mathbf{r}_{1}-U_{1} \mathbf{r}_{u}-V_{1} \mathbf{r}_{v} .
$$

This enables determination of $t(u, v)$ and $\mathbf{r}(u, v)$, similar to the forward problem. Here too the vector $\mathbf{w}$ is collinear to the normal to the surface (Eq. 19) and satisfies $\mathbf{w}=k \mathbf{n}$. Let the rate of change of $s_{1}$ be a constant, $s_{10}$. The ODEs containing the unknowns $\left(s_{1}, s_{2}, \tau_{1}, \tau_{2}, \mathbf{r}_{u}, \mathbf{r}_{v}, \rho\right)$ can be written as follows:

$$
\begin{gathered}
s_{1}=\dot{s}_{10} t, \dot{\tau}_{1}=\dot{s}_{10} \mathbf{c}_{1} \cdot \mathbf{a}_{1}, \dot{s}_{2}=-\frac{k \mathbf{f}_{2} \cdot \mathbf{b}_{2}}{\mathbf{e}_{2} \cdot \mathbf{b}_{2}+\mathbf{c}_{2} \cdot\left[\left(\mathbf{c}_{2} \cdot \mathbf{a}_{2}\right) \mathbf{d}_{2}+\mathbf{G}_{2} \cdot \mathbf{c}_{2}\right]}, \\
\dot{\tau}_{2}=\dot{s}_{2} \mathbf{c}_{2} \cdot \mathbf{a}_{2}, k=-\frac{\mathbf{e}_{1} \cdot \mathbf{b}_{1}+\mathbf{c}_{1} \cdot\left[\left(\mathbf{c}_{1} \cdot \mathbf{a}_{1}\right) \mathbf{d}_{1}+\mathbf{G}_{1} \cdot \mathbf{c}_{1}\right]}{\mathbf{f}_{1} \cdot \mathbf{b}_{1}} \dot{s}_{10}, \dot{\mathbf{r}}_{u}=k h_{u} \mathbf{n}, \\
\dot{\mathbf{r}}_{v}=k h_{v} \mathbf{n}, \dot{\rho}=-k \mathbf{n}, h_{u}=\frac{v_{2}-v_{1}}{u_{1} v_{2}-u_{2} v_{1}}, h_{v}=\frac{u_{1}-u_{2}}{u_{1} v_{2}-u_{2} v_{1}}, \\
\mathbf{a}_{i}\left(\tau_{i}, \mathbf{r}_{i}\right)=\frac{x_{i}^{\prime} \nabla F_{x}\left(\mathbf{r}_{1}\right)+y_{i}^{\prime} \nabla F_{y}\left(\mathbf{r}_{i}\right)}{x_{i}^{\prime 2}+y_{i}^{\prime 2}}, \mathbf{b}_{i}\left(\tau_{i}, \mathbf{r}_{i}\right)=y_{i}^{\prime} \nabla F_{x}\left(\mathbf{r}_{i}\right)-x_{i}^{\prime} \nabla F_{y}\left(\mathbf{r}_{i}\right), \\
\mathbf{c}_{i}\left(s_{i}, \mathbf{r}_{u}, \mathbf{r}_{v}\right)=u_{i}^{\prime} \mathbf{r}_{u}+v_{i}^{\prime} \mathbf{r}_{v}, \mathbf{d}_{i}=y_{i}^{\prime \prime} \nabla F_{x}\left(\mathbf{r}_{i}\right)-x_{i}^{\prime \prime} \nabla F_{y}\left(\mathbf{r}_{i}\right), \quad \mathbf{e}_{i}=u_{i}^{\prime \prime} \mathbf{r}_{u}+v_{i}^{\prime \prime} \mathbf{r}_{v}, \\
\mathbf{f}_{i}=\left(u_{i}^{\prime} h_{u}+v_{i}^{\prime} h_{v}\right) \mathbf{n}, \mathbf{G}_{i}=y_{i}^{\prime} \nabla \nabla F_{x}\left(\mathbf{r}_{i}\right)-x_{i}^{\prime} \nabla \nabla F_{y}\left(\mathbf{r}_{i}\right) .
\end{gathered}
$$


To start the integration of the inverse problem, we need initial conditions for $\left(s_{1}, s_{2}, \tau_{1}\right.$, $\left.\tau_{2}, \mathbf{r}_{u}, \mathbf{r}_{v}, \rho\right)$.

Solution to the Boundary Value Problem: While the equation above can be solved for a general camera model, we will consider the simple orthographic case here. We can show these initial values here are:

$$
\begin{aligned}
t_{0} & =s_{10}=s_{20}=\tau_{10}=\tau_{20}=0, \mathbf{r}_{10}=\mathbf{r}_{20}=\mathbf{r}_{0}, \\
u_{10} & =u_{20}=u_{0}, \quad v_{10}=v_{20}=v_{0}, x_{10}=x_{20}=F_{x}\left(\mathbf{r}_{0}\right), y_{10}=y_{20}=F_{y}\left(\mathbf{r}_{0}\right),
\end{aligned}
$$

and for the starting point in $3 \mathrm{D}, \mathbf{r}_{0}=\mathbf{r}_{0}\left(x_{0}, y_{0}, z_{0}\right)$ where $z_{0}$ is some free parameter in the orthographic case. Note also that at the initial point the formulae for $h_{u}$ and $h_{v}$

$$
h_{u}=\frac{v_{2}-v_{1}}{u_{1} v_{2}-u_{2} v_{1}}, \quad h_{v}=\frac{u_{1}-u_{2}}{u_{1} v_{2}-u_{2} v_{1}} .
$$

are not acceptable, since the numerators and denominators are zero. However, we can find $h_{u 0}$ and $h_{v 0}$ from

$$
u_{0} h_{u 0}+v_{0} h_{v 0}=1, \quad \dot{s}_{10}\left(u_{10}^{\prime} h_{u 0}+v_{10}^{\prime} h_{v 0}\right)=\dot{s}_{20}\left(u_{20}^{\prime} h_{u 0}+v_{20}^{\prime} h_{v 0}\right) .
$$

The solution of this linear system specifies $h_{u 0}$ and $h_{v 0}$ as a function of $\dot{s}_{20}$, which can be estimated from the free parameter, and is in fact one of the Euler angles $\gamma_{0}$. Recalling that $\left(\mathbf{r}_{u}, \mathbf{r}_{v}, \mathbf{r}_{u} \times \mathbf{r}_{v}\right)$ is a right-handed basis, we can rotate the reference frame of the world coordinates by Euler angles $\left(\alpha_{0}, \beta_{0}, \gamma_{0}\right)$ so that we have $\mathbf{r}_{u 0}=(1,0,0), \mathbf{r}_{v 0}=$ $(0,1,0)$. Further:

$$
\begin{gathered}
\dot{s}_{10} \mathbf{e}_{10} \cdot \mathbf{b}_{10}+k_{0} \mathbf{f}_{10} \cdot \mathbf{b}_{10}+\dot{s}_{10} \mathbf{c}_{10} \cdot\left[\left(\mathbf{c}_{10} \cdot \mathbf{a}_{10}\right) \mathbf{d}_{10}+\mathbf{G}_{10} \cdot \mathbf{c}_{10}\right]=0, \\
\dot{s}_{20} \mathbf{e}_{20} \cdot \mathbf{b}_{20}+k_{0} \mathbf{f}_{20} \cdot \mathbf{b}_{20}+\dot{s}_{20} \mathbf{c}_{20} \cdot\left[\left(\mathbf{c}_{20} \cdot \mathbf{a}_{20}\right) \mathbf{d}_{20}+\mathbf{G}_{20} \cdot \mathbf{c}_{20}\right]=0, \\
\mathbf{c}_{10} \cdot \mathbf{b}_{10}=0, \mathbf{c}_{20} \cdot \mathbf{b}_{20}=0 .
\end{gathered}
$$

These 4 relations can be treated as equations relating the 10 unknowns $k_{0}, \mathbf{r}_{u 0}, \mathbf{r}_{v 0}, \mathbf{n}_{0}$ $\left(\mathbf{r}_{u 0}, \mathbf{r}_{v 0}\right.$ and $\mathbf{n}_{0}$ are $3 \mathrm{D}$ vectors). Also $\mathbf{r}_{u 0}, \mathbf{r}_{v 0}$, and $\mathbf{n}_{0}$ form an orthonormal basis, which therefore can be completely described by the three Euler angles $\left(\alpha_{0}, \beta_{0}, \gamma_{0}\right)$ :

$$
\mathbf{r}_{u 0}=Q_{0}\left(\begin{array}{l}
1 \\
0 \\
0
\end{array}\right), \quad \mathbf{r}_{v 0}=Q_{0}\left(\begin{array}{l}
0 \\
1 \\
0
\end{array}\right), \quad \mathbf{n}_{0}=Q_{0}\left(\begin{array}{l}
0 \\
0 \\
1
\end{array}\right),
$$

where $Q_{0}$ is the Euler rotation matrix. This shows that $\mathbf{r}_{u 0}, \mathbf{r}_{v 0}$, and $\mathbf{n}_{0}$ a three-parameter set depending on $\left(\alpha_{0}, \beta_{0}, \gamma_{0}\right)$. Thus the relations Eq. (37) can be treated as 4 equations with respect to the unknowns $k_{0}, \alpha_{0}, \beta_{0}, \gamma_{0}$, for given $\dot{s}_{20}$ or $k_{0}, \alpha_{0}, \beta_{0}, \dot{s}_{20}$ for given $\gamma_{0}$, and can be solved. Then

$$
\rho_{0}=\mathbf{r}_{0}-u_{0} \mathbf{r}_{u 0}-v_{0} \mathbf{r}_{v 0}
$$

determines $\rho_{0}$ as soon as $\mathbf{r}_{u 0}, \mathbf{r}_{v 0}$, and $\mathbf{r}_{0}$ are specified. Furthermore, we can reduce the four equations above to one nonlinear equation, whose roots can be determined by conventional numerical methods [17]. 
We found that this equation has two solutions, and so the Euler angles have four possible values. By choosing the free parameter $\gamma_{0}$ (Orthographic case), we can set all the initial conditions needed for the inverse problem. The challenge is to get the best estimate of $\gamma_{0}$ so that the boundary condition specifying correspondence points (such as the corners) is achieved. This is called the shooting method. We do this by minimizing a cost function $J$ :

$$
J=\underset{\gamma_{0}}{\arg \min }\left\|\left(x_{e}, y_{e}\right)-\mathbf{F}\left(\mathbf{r}\left(t_{\max } ; \gamma_{0}, \Gamma_{1}, \Gamma_{2}, \Gamma_{1}^{\prime}, \Gamma_{2}^{\prime}\right)\right)\right\|,
$$

where $\left(x_{e}, y_{e}\right)$ is the image coordinates of the 3D surface ending point $\left(X_{e}, Y_{e}, Z_{e}\right)$ and $\mathbf{r}\left(t_{\max } ; \gamma_{0}, \Gamma_{1}, \Gamma_{2}, \Gamma_{1}^{\prime}, \Gamma_{2}^{\prime}\right)$ is the last step of the $3 \mathrm{D}$ structure solution and $\mathbf{F}$ is the camera model function. It is clear that $\mathbf{F}\left(\mathbf{r}\left(t_{\max } ; \gamma_{0}, \Gamma_{1}, \Gamma_{2}, \Gamma_{1}^{\prime}, \Gamma_{2}^{\prime}\right)\right)$ is the ending point of 3D surface calculated by the ODE solver. Therefore, we change the free parameter $\gamma_{0}$ until we can hit the ending corner or are within a specified tolerance of the ending point in the image plane. If the number of the correspondence points on the edge available exceeds the number of shooting parameters (say the 4 corners) a least-square approach can be used.

Ambiguities: As stated in the inverse problem, the method relies on the boundary information of the patch in the image plane. So, since some deformations can lead us to the same images of the boundary, we have ambiguities. In these cases we need to extract other useful cues such as texture or shading to resolve the ambiguities. This is the subject for future work.

\section{Discussion and Results}

\subsection{Simple Validation of the Forward Problem}

The purpose of this paper is to present and validate the new method. For this purpose we implemented the solution in algorithms. In the validation stage, we compared the results for warping to a $3 \mathrm{D}$ curve with the following analytical solution corresponding to a cylindrical surface

$$
X=u-u_{\min }, Y=N \cos \varphi(v), Z=N \sin \varphi(v), \varphi(v)=v / N .
$$

To reproduce this surface we started our algorithm for warping with a 3D curve with the condition that in the $(u, v)$-plane the curve is a straight line, $u=u_{\min }$, and the fact that the corresponding $3 \mathrm{D}$ curve is

$$
X(t)=0, Y(t)=N \cos \varphi(t), Z(t)=N \sin \varphi(t) .
$$

For this surface we have the initial conditions for integration as $\mathbf{r}_{u 0}=(-1,0,0)$, $\mathbf{r}_{v 0}=\left(0,-\sin \varphi_{0}, \cos \varphi_{0}\right)$ with $\varphi_{0}=v_{\min } / N$. We integrated the forward problem Eq. (29) numerically using an ODE solver from MATLAB, which was based on the $4^{\text {th }}$ order Runge-Kutta method. The results were identical to the analytical solution within the tolerance specified to the solver. We also checked that solution (30) is correct.

\subsection{Forward Problem: Implementation Issues and Results}

After initial tests we used the method of warping with 3D curves for generation of more complex applicable surfaces. The tests were performed both by straightforward numerical integration of ODE's (29) and using the analytical solution for rectangular pathces 
(30). Both methods showed accurate and consistent results. To generate an example curve $\mathbf{R}(t)$ parametrized naturally, we specified another function $\widetilde{\mathbf{R}}(\theta)$ where $\theta$ is an arbitrary parameter and then used transform

$$
\mathbf{R}(t)=\widetilde{\mathbf{R}}(\theta), \quad \frac{d t}{d \theta}=\left|\frac{d \widetilde{\mathbf{R}}(\theta)}{d \theta}\right|,
$$

which provides $|\dot{\mathbf{R}}|=1$, and guarantees that $t$ is the natural parameter. The function $\widetilde{\mathbf{R}}(\theta)$ used in tests was

$$
\widetilde{\mathbf{R}}(\theta)=(P(\theta), N \cos \theta, N \sin \theta), \quad P(\theta)=a_{1} \theta+a_{2} \theta^{2}+a_{3} \theta^{3}+a_{4} \theta^{4},
$$

and some other than polynomial dependencies $P(\theta)$ were tested as well. One of the examples of image warping with a $3 \mathrm{D}$ curve is presented in Figure 5.

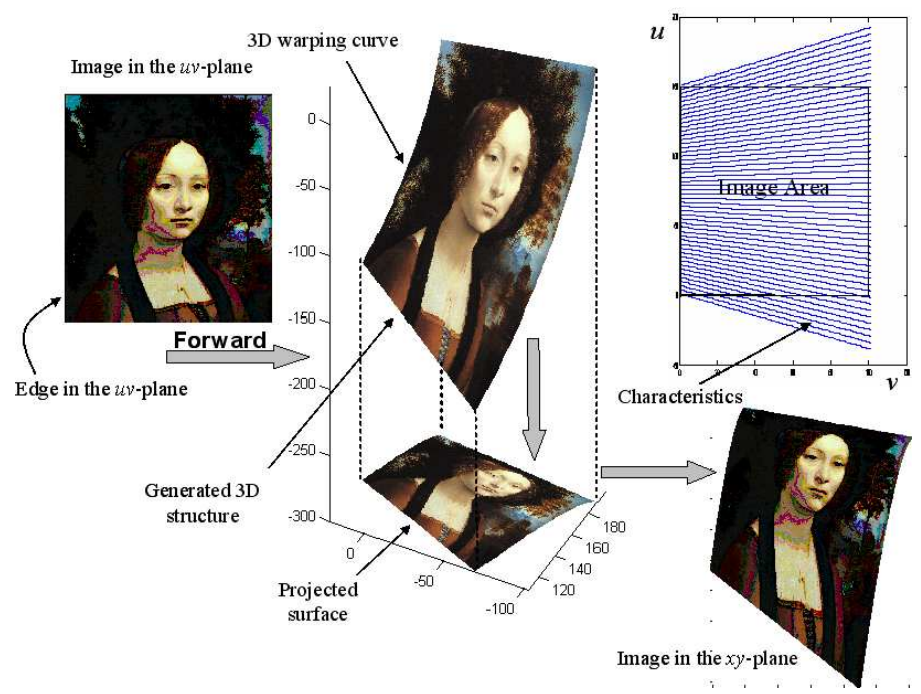

Fig. 5. 'Forward' problem: given a plane sheet of paper, and a smooth 3-D open curve in Cartesian $X Y Z$ space. Our goal is to bend the paper so that one edge conforms to the specified curve. Using the analytical integration of the differential geometric equations specifying applicability we are able to achieve this. We can also achieve the same result not only for the straight line edge, but for an arbitrary 2-D curve in the $u v$-plane. The picture shown are actual computations.

For this case the boundary curve were selected in the form (43), with parameters $N=200, a_{1}=20, a_{2}=10, a_{3}=10, a_{4}=-10$ and we used Eqs (31) and (34) to generate the $3 \mathrm{D}$ structure and characteristics. In this example the characteristics for this surface are not parallel, which is clearly seen from the graph in the upper right corner of Fig. 5. The image of the portrait of Ginevra dé Bencia by Leonardo da Vinci, was fit into a rectangle in the $u v$-plane and warped with the generated surface. Further its orthographic projection was produced using pixel-by-pixel mapping of the obtained transform from the $(u, v)$ to the $(x, y)$. These pictures are also shown in Figure 5. 


\subsection{Inverse Problem: Implementation Issues and Results}

To check the validity of the unwarping procedure, we ran the 2D unwarping problem with synthetic input data on the patch boundaries and corner correspondence points obtained by the warping procedure. The output of the solver providing $h_{u}, h_{v}, \mathbf{r}_{u}, \mathbf{r}_{v}$, and $\rho$ as functions of $t$ coincided with these functions obtained by the 3D curve warping program within the tolerance specified for the ODE solver. The unwarped pixel-by-pixel images are shown in Figure 6 as the end point of the unwarping process in the $x y$ plane. We ran the algorithm for small fonts. The original image has the same font size everywhere and with the forward algorithm we warp the image. The unwarped image has uniform font size everywhere, lines are parallel and right angles are preserved. The output is noisy at the top of the output image, since in the image this information was lost. We make the following remarks about the implementation of the inverse problem:

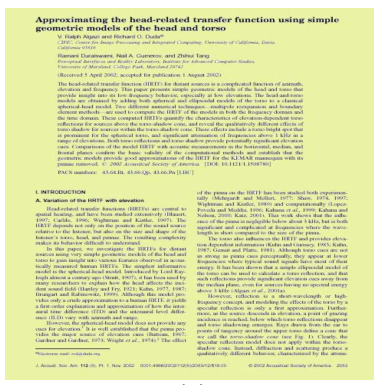

(a)

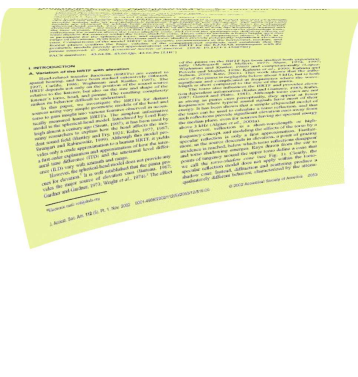

(b)

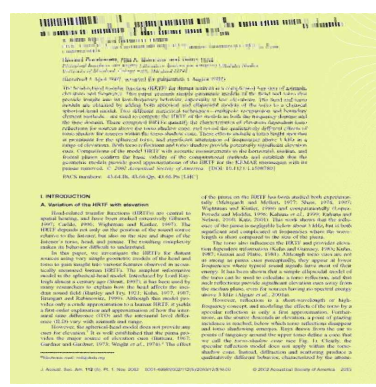

(c)

Fig. 6. Inverse Problem for small font: a) original image b) warped by the forward $\tilde{\mathbf{R}}(\theta)=$ $\left(a \theta\left(b-\theta^{3}\right), N \cos \theta, N \sin \theta\right)$ where $a=10, b=2, N=200$ c) unwarped by the inverse problem

Global Parametrization: In the inverse problem, we march the ODE's with respect to the bounding contours in $u v$-plane and $x y$-plane. Therefore, for simplicity and modularity, we use a global parameter $\eta$ for bounding contours that runs from $\eta$ in $[0,1]$ on the first boundary to $\eta=[3,4]$ on the last. This parameterization gives us a simple and exact way of tracking the edges at the boundary contours and the correspondence between them.

ODE solver: To solve the ODE, we applied the Runge-Kutta 4th and 5th order in MATLAB, except for the last edge of the ODE, where the problem was computationally stiff. For this, we solved the ODE by Gear's method [17].

Automatic Corner Detection by ODE solver: We need the corners in the image plane for the boundary of the patch to solve the inverse problem. As stated, the global natural parameterization of the curve in image plane, gives us an easy and reliable feature for corner detection. Basically, the corner is reached when $s_{2}$ and $\tau_{2}$ (global parameters of $\Gamma_{2}^{\prime}$ and $\Gamma_{2}$ ) are 1,2 and 3, respectively.

\section{Conclusion and Future Work}

This paper presents, to our knowledge, the first occasion that differential geometry has been used quantitatively in the recovery of structure from images. A theory and method 
for warping and unwarping images for applicable surfaces based on patch boundary information and solution of nonlinear PDEs of differential geometry was developed. The method is fast, accurate and correspondence free (except for a few boundary points).

We see many useful applications of this method for virtual reality simulations, computer vision, and graphics; e.g. 3D reconstruction, animation, object classification, OCR, etc. While the purpose of this study was developing and testing of the method itself, ongoing work is related both to theoretical studies and to development of practical algorithms. This includes more detailed studies of the properties of the obtained equations, problems of camera calibration, boundary extraction, sensitivity analysis, efficient minimization procedures, and unwarping of images acquired by a camera, where our particular interest is in undoing the curl distortion of pages with printed text.

\section{References}

1. M. S. Brown and W. B. Seales. Document restoration using 3D shape: A general deskewing algorithm for arbitrarily warped documents In ICCV 2001, 2001

2. P. Clark and M. Mirmehdi. Estimating the orientation and recovery of text planes in a single image In Proceedings of the British Machine Vision Conference, 2001

3. D.A. Forsyth and J. Ponce. Computer Vision: A Modern Approach Prentice Hall, 2003

4. G.B. Whitham, Linear and Nonlinear Waves, New-York: Wiley, 1974

5. R. Hartley and A. Zissermann. Multiple View Geometry in Computer Vision In Cambridge Press, 2000

6. J. Garding. Surface orientation and curvature from differential texture distortion In International Conference on Computer Vision, ICCV, 1995

7. J.J. Koenderink. What Does the Occluding Contour Tell us About Solid Shape Perception, 13: 321-330, 1984

8. J.J. Koenderink, Solid Shape, MIT Press, 1990.

9. G. A. Korn and T.M. Korn. Mathematical Handbook for scientists and engineers In Dover Publications, Inc., 1968

10. Y. L. Kergosien, H. Gotoda and T. L. Kunii. Bending and creasing virtual paper In IEEE Computer graphics and applications, Vol. 14, No. 1, pp 40-48, 1994

11. T. Kanungo, R. Haralick, and I. Phillips. Nonlinear Local and Global Document Degradation Models In Int'l. J. of Imaging Systems and Tech., Vol. 5, No. 4, pp 220-230, 1994

12. D. Liebowitz and A. Zisserman. Metric rectification for perspective images of planes In IEEE Computer Vision and Pattern Recognition Conference, pp 482-488, 1998

13. M. A. Penna. Non-rigid Motion Analysis: Isometric Motion In CVGIP: Image Understanding, Vol. 56, No. 3, pp 366-380, 1992.

14. M. Do Cormo. Differential Geometry of Curves and Surfaces. Prentice Hall, 1976

15. M. Pilu. Extraction of illusory linear clues in perspectively skewed documents In IEEE Computer Vision and Pattern Recognition Conference, 2001

16. M. Pilu. Undoing Page Curl Distortion Using Applicable Surfaces In Proc. IEEE Conf Comouter Vision Pattern Recognition, 2001

17. W.H. Press, S.A. Teukolsky, W.T. Vetterling and B. P. Flannery. Numerical Recipes in C, Cambridge University Press, 1993

18. R.I. Hartley. Theory and practice of projection rectification In International Journal of Computer Vision, Vol.2, No. 35, pp 1-16, 1999

19. Y. You, J. Lee and Ch. Chen. Determining location and orientation of a labeled cylinder using point pair estimation algorithm In International Journal of Pattern Recognition and Artificial Intelligence, Vol. 8, No. 1, pp 351-371, 1994 